\title{
INTERVENCIÓN DE TERAPIA OCUPACIONAL EN NIÑOS CON DIFICULTADES DE PROCESAMIENTO SENSORIAL: UN ESTUDIO DE CASO MÚLTIPLE EN UNA ESCUELA EN PARIS.
}

\author{
OCCUPATIONAL THERAPY INTERVENTION FOR CHILDREN WITH CHALLENGES IN \\ SENSORY PROCESSING: A MULTIPLE CASE STUDY FROM A SCHOOL IN PARIS
}

\section{Clara Zambruno ${ }^{1}$}

\begin{abstract}
RESUMEN
Propósito: El principal objetivo de este estudio fue describir el proceso y evaluar la eficacia de terapia ocupacional con abordaje de integración sensorial para 3 niños con dificultades de procesamiento sensorial, con el fin de ayudar a desarrollar modelos de practica apropiados en planificación e implementación de la intervención de terapia ocupacional.

Método: Se utilizó un diseño de estudio de casos múltiples. Todos los participantes eran estudiantes de la misma escuela y fueron remitidos a terapia ocupacional debido a dificultades en escritura, torpeza motora y participación en clase. Los participantes recibieron sesiones individuales de 45 minutos de duración. La duración media de la intervención fue de 27 sesiones realizadas una vez por semana en el transcurso del año lectivo. Los niños fueron evaluados antes y después de la intervención con entrevistas a padres y maestros, observaciones clínicas y pruebas estandarizadas.

Resultados: Luego de la intervención se observaron mejores resultados en habilidades de desempeño de las evaluaciones estandarizadas Bruininks-Oseretsky Test of Motor Proficiency, Segunda Edición (BOT $\left.{ }^{\mathrm{TM}}-2\right)$ y Prueba de Desarrollo de la Percepción Visual - Tercera Edición (DTVP-3). Además, se observó mejora en la calidad de la escritura y la participación en los juegos de motricidad gruesa en todos los niños.

Conclusión: Los resultados de este estudio se adhieren a la literatura en el sentido de que la terapia ocupacional bajo un marco de integración sensorial parece ser un método efectivo para mejorar las habilidades de desempeño y la participación funcional de los niños con desafíos de procesamiento sensorial.
\end{abstract}

\section{PALABRAS CLAVE}

Terapia Ocupacional; Niño; Instituciones Académicas; Desempeño Psicomotor; Trastornos de la sensación.

\begin{abstract}
Purpose: The main purpose of this study was to describe the process and evaluate the effectiveness of occupational therapy under a sensory integration framework for 3 children with sensory processing challenges, in order to help develop models of good practice in planning and implementation of occupational therapy intervention.
\end{abstract}

1 Master of Arts in Occupational Therapy. University of Southern California. Los Angeles, Estados Unidos. Licenciada en Terapia Ocupacional. Universidad del Salvador. Buenos Aires, Argentina. Certificación en Integración Sensorial. Universidad del Sur de California 2 Rue Adolphe Lalyre, 9240o, Courbevoie, Francia. clarazambruno@gmail.com $+33768540648$ 
Method: A Multiple Case Study design was utilized. All participants were students at the same school and were referred to occupational therapy because of concerns with handwriting, clumsiness and class participation. Participants received individual, 45 minutes long intervention sessions. The mean length of intervention was 27 sessions delivered t time per week over the course of the academicyear. Children were evaluated before and after intervention with parent/teacher interviews, clinical observations and standardized tests.

Results: After the intervention all participants obtained higher scores for performance skills in the standardized tests Bruininks-Oseretsky Test ofMotor Proficiency, Second Edición (BOT ${ }^{T M}$-2) and Developmental Test of Visual Perception - Third Edition (DTVP-3). Furthermore, improved handwriting quality and participation on gross motor games was reported for all participants.

Conclusion: The results of this study adhere to the literature in that occupational therapy under a sensory integration framework seems to be an effective method for enhancing performance skills and functional participation of children with sensory processing challenges.

\section{KEYWORDS}

Occupational Therapy; Child; Schools; Psychomotor Performance; Sensation Disorders.

Recepcionado: 27/08/2020

Aceptado: $27 / 11 / 2020$

\section{INTRODUCCIÓN}

La literatura sugiere que entre el 10\% y el 55\% de los niños sin discapacidad diagnosticada se enfrentan a desafíos en el procesamiento sensorial (Watling, Kuhaneck, Parham, \& Schaaf, 2018). Estas dificultades suelen derivar en conductas problemáticas, baja autoestima, pobres habilidades sociales y bajo rendimiento académico (Ayres, 1972). La literatura emergente sostiene, asimismo que los niños con dificultades de procesamiento sensorial, con y sin discapacidad, exhiben dificultades de desempeño funcional en ocupaciones de la infancia (Koenig \& Rudney, 2010); (Pfeiffer, May-Benson, \& Bodison, 2018). Por otro lado, la evidencia sostiene el uso de diferentes enfoques dentro del dominio y proceso de terapia ocupacional para la intervención con esta población y en una variedad de ambientes, incluyendo colegios privados y públicos. (Watling et al., 2018)(Smith-Roley, Clark, \& Bissell, 2003)(Smith-Roley, Bissell, \& Clark, 2009)(Reynolds, Glennon, Ausderau, Bendixen, Miller, Pfeiffer, Watling, Wilkinson \& Bodison, 2017)

Sin embargo, si bien existe evidencia de cómo los profesionales de la terapia ocupacional evalúan la capacidad de los estudiantes para participar en la escuela y pueden proporcionar servicios para mejorar el aprendizaje, el rendimiento académico y la participación (Grajo,
Candler, \& Sarafian, 202O) (Clark, Watling, Parham, \& Schaaf, 2019), en algunos países, como Francia, contar con una terapista ocupacional de planta en los colegios no es una práctica común. Asimismo, la relación entre dificultades de procesamiento sensorial y problemas de desempeño es poco conocida y difundida en el ámbito escolar. Sumado a ello, los niños con problemas de procesamiento sensorial son frecuentemente pasados por alto o sus dificultades son malinterpretadas como problemas de comportamiento y no reciben el tratamiento adecuado.

El siguiente estudio de carácter cualitativo y descriptivo tiene como objetivo explorar el proceso y evaluar la eficacia de la Terapia Ocupacional bajo el abordaje de integración sensorial para tres niños con problemas de procesamiento sensorial, en contexto escolar real. Estos niños fueron elegidos, en parte, debido a que fueron sus maestras quienes identificaron dificultades que podrían estar relacionadas a problemas de procesamiento sensorial y consultaron a la terapista ocupacional de planta. El autor cree firmemente que un mayor conocimiento y entendimiento tanto por padres como maestros; así como contar con la posibilidad de consultar de forma inmediata con un profesional, son componentes claves para la identificación de problemas de procesamiento sensorial, posibilitando la intervención temprana y la prevención de mayores dificultades futuras. 


\section{MÉTOdo}

Muestra: Dos niños y una niña, estudiantes de la misma escuela primaria en Paris, Francia, fueron incluidos en el estudio. Dicha escuela cuenta con una terapista ocupacional de planta y un gimnasio terapéutico donde se realizaron las evaluaciones e intervenciones.

Los casos fueron elegidos considerando similitud entre ellos. Las maestras de los niños fueron quienes consultaron al profesional y solicitaron una evaluación debido a preocupaciones relacionadas principalmente con la calidad y cantidad de escritura, dificultades en destrezas motoras y problemas de atención y participación en clase. Debido a que en ningún caso había discapacidad intelectual o de desarrollo asociada, eran candidatos apropiados para el servicio directo usando Intervención de Integración Sensorial (Watling et al., 2018).

Recolección de datos: Luego de una breve observación en clase y conversación con las maestras respectivas, se determinó la necesidad de realizar una evaluación exhaustiva de terapia ocupacional para determinar si las dificultades de desempeño funcional podían estar relacionadas a problemáticas en el procesamiento sensorial. Los niños fueron evaluados en 3 sesiones de 45 minutos en el gimnasio terapéutico de la escuela. Se utilizaron los siguientes instrumentos tanto para la evaluación como la re-evaluación con el fin de abordar aspectos de desempeño funcional, componentes de desempeño y cambios en el procesamiento sensorial de los niños.

- Perfil Ocupacional: se utilizó el cuestionario "Antecedentes y Perfil Ocupacional" (Schaaf \& SmithRoley, 2006) y se completó con una entrevista no estructurada a padres y maestros.

- Sensory Processing Measure(SPM), Escuela y Hogar (Parham \& Ecker, 2007):es un cuestionario para padres, cuidadores y maestros con el fin de obtener información clave sobre el comportamiento sensoriomotor y procesamiento sensorial de niños de 5 a 12 años. Arroja puntuaciones T para los sistemas sensoriales, la praxis y la participación social.

- Bruininks-Oseretsky Test of Motor Proficiency, Segunda Edición $\left(\mathrm{BOT}^{\mathrm{TM}}-2\right)$ (Bruininks \& Bruininks, 2005): es una evaluación estandarizada, para individuos de 4 a 21 años, administrado individualmente que utiliza diferentes actividades para medir el desempeño motor en áreas funcionales de estabilidad, movilidad, fuerza, coordinación y manipulación de objetos.

- $\quad$ Prueba de Desarrollo de la Percepción Visual - Tercera Edición (DTVP-3) (Hammill, Pearson, \& Voress, 2013)es una evaluación estandarizada diseñada para identificar la presencia y el grado de dificultades de percepción visual y visual-motoras en niños de 4 a 12 años.

- Observaciones Clínicas de Procesamiento Sensorial (Blanche, 2010)

- Observaciones no estructuradas en clase y en el gimnasio terapéutico.

Los padres de los niños firmaron su consentimiento informado en el cual autorizaron a realizar la evaluación y tratamiento de terapia ocupacional, así como la utilización de esta información con fines de investigación o educacionales. Asimismo, los directivos de la institución educativa fueron informados y dieron su consentimiento para la presentación de este estudio.

\section{Evaluación de Terapia Ocupacional}

\section{Caso Ana}

\section{Perfil Ocupacional}

Ana era una niña de 7 años y 7 meses al momento de la evaluación. Vivía con sus padres y cursaba el segundo grado en una escuela de Paris. Sus padres y maestros la describían como una niña cariñosa y amigable con buenas habilidades de lenguaje oral y lectura. Disfrutaba mucho de jugar con sus peluches y de leer.

Ana nació luego de un embarazo y parto sin complicaciones, cumplió las pautas iniciales de desarrollo madurativo a edades esperadas, y no tenía antecedentes médicos relevantes. 
TABLA 1 EVALUACIÓN DE COMPONENTES DE DESEMPEÑO

\begin{tabular}{lll}
\hline Evaluación & \multicolumn{1}{c}{ Área de dificultad } & \multicolumn{1}{c}{ Desempeño } \\
\hline \multirow{2}{*}{ SPM Hogar } & Visión & Algunos Problemas (T=61) \\
& Tacto & Algunos Problemas (T=64) \\
& Conciencia corporal & Algunos Problemas (T=67) \\
& Movimiento Y equilibrio & Algunos Problemas (T=68) \\
& Planeamiento motor e ideación & Algunos Problemas (T=69) \\
\hline SPM Escuela & - & - \\
\hline & Precisión motora fina & Debajo del promedio (SS=9) \\
& Destreza manual & Debajo del promedio (SS=10) \\
& Coordinación de E.S & Debajo del promedio (SS=7) \\
& Fuerza & Debajo del promedio (SS=7) \\
BOT-2 & Velocidad de carrera y agilidad & Debajo del promedio (SS=6) \\
& Coordinación Bilateral & Muy por debajo del promedio \\
& Equilibrio & (SS=5) \\
& & Muy por debajo del promedio \\
& Coordinación ojo-mano & (SS=5) \\
\hline Observaciones Clínicas & Febajo del promedio (SS=7) \\
& Cigura-fondo & Debajo del promedio (SS=7) \\
& Diadococinesis & Debajo del promedio (SS=7) \\
\hline
\end{tabular}

Nota:

$T$ = Puntuaciones T. De 50 a 59 indican respuestas de comportamiento típico, y por encima de 59, a mayor puntaje mayores dificultades.

SS $=$ Scale Scores

En el BOT-2: Muy por debajo del promedio <5; Debajo del promedio 6 - 10; Promedio 11 - 19.

En el DTVP-3: Pobre 4 - 5; Debajo del promedio 6 - 7; Promedio 8 - 12.

E.S $=$ Extremidades Superiores

Relación entre resultados de la evaluación y desempeño ocupacional

Se observaron dificultades en la legibilidad y desprolijidad de la escritura, y en la realización de proyectos de arte, lo que podría estar relacionado al pobre procesamiento táctil y propioceptivo impactando negativamente el planeamiento, coordinación y destreza motora fina. Asimismo, la niña presentaba pobre procesamiento vestibular-ocular y dificultades en la percepción visual lo que podría influir en aspectos de la escritura como el posicionamiento y orientación en la hoja. Presentaba nivel de alerta y atención bajo que podría relacionarse a una baja responsividad sensorial, 
sobre todo a inputs vestibulares que inciden en la regulación del alerta. La niña elegía juegos sedentarios, se reusaba a participar en deportes de equipo y experimentaba ansiedad durante fiestas de cumpleaños que involucraban actividades de motricidad gruesa, como escalar. Esto se debía a su pobre conciencia corporal, control postural, coordinación motora bilateral y planeamiento motor, a causa, probablemente de su bajo procesamiento táctil, propioceptivo y vestibular. Ana no notaba cuando tenía restos de comida en su boca o cuando su ropa estaba desalineada lo que podría relacionarse a su dificultad para localizar y discriminar el input táctil. Ponía demasiada comida en su boca, masticaba objetos no comestibles, su pelo, su ropa y succionaba su pulgar. Estas conductas podrían relacionarse a un bajo registro táctil y propioceptivo en el área oral y a una búsqueda activa de dicha información faltante. Sin embargo, también podrían estar relacionadas a técnicas de auto-regulación ante situaciones de stress o dificultad. Ana presentaba dificultades para el manejo de las emociones, ocasionalmente intentar responder a las demandas del contexto se volvía muy frústrate lo que daba lugar a arrebatos emocionales y enfados principalmente en el hogar.

\section{Caso Benjamín}

\section{Perfil Ocupacional}

Benjamín era un niño de 7 años y 5 meses al momento de la evaluación. Vivía con sus padres y su hermana menor, y cursaba el segundo grado en una escuela de Paris. Sus padres y maestros lo describían como una niño inteligente, amable e imaginativo con buenas habilidades de razonamiento y comprensión lectora. Una evaluación neuropsicológica indicó un IQ en el rango superior.

Benjamín nació tras una cesárea de emergencia a las 39 semanas de gestación. Requirió oxígeno al momento del nacimiento y permaneció un breve período en la NICU, sin embargo, no se reportaron grandes consecuencias. Benjamín tenía antecedentes de otitis crónica y tenía implante de tubos. Por otro lado, las pautas iniciales de desarrollo madurativo fueron alcanzadas a edades esperadas, y no tenía antecedentes médicos relevantes adicionales.

TABLA 2. EVALUACIÓN DE COMPONENTES DE DESEMPEÑO

\begin{tabular}{|c|c|c|}
\hline Evaluación & Área de dificultad & Desempeño \\
\hline SPM Hogar & Conciencia corporal & Algunos Problemas $(\mathrm{T}=60)$ \\
\hline \multirow{3}{*}{ SPM Escuela } & Participación Social & Algunos Problemas ( $\mathrm{T}=62)$ \\
\hline & Visión & Algunos Problemas ( $\mathrm{T}=62)$ \\
\hline & Audición & Algunos Problemas $(\mathrm{T}=60)$ \\
\hline \multirow{4}{*}{ BOT-2 } & Coordinación de E.S & Debajo del promedio $(\mathrm{SS}=8)$ \\
\hline & Fuerza & Debajo del promedio $(S S=10)$ \\
\hline & Coordinación Bilateral & Debajo del promedio (SS=7) \\
\hline & Equilibrio & Debajo del promedio (SS=6) \\
\hline DTVP-3 & Coordinación ojo-mano & Pobre $(S S=4)$ \\
\hline \multirow{5}{*}{ Observaciones Clínicas } & Diadococinesis & \\
\hline & Toque secuencial de dedos & \\
\hline & Movimientos Oculares & \\
\hline & Posiciones anti-gravitatorias & \\
\hline & Estabilidad articular proximal & \\
\hline Nota: Se utilizaron las mismas a & nes que en la Tabla 1 & \\
\hline
\end{tabular}




\section{Relación entre resultados de la evaluación y desem-} peño ocupacional

Benjamín presentaba dificultades para permanecer sentado y sostener su atención, se movía inquietamente en la silla y adoptaba una postura encorvada. Asimismo, era desordenado al comer y derramaba comida con frecuencia. Esto podría relacionarse a un ineficiente control postural debido a problemas de procesamiento propioceptivo y vestibular. Por otro lado, poseía buenas habilidades de motricidad fina y percepción visual, componentes claves para la escritura. Sin embargo, su escritura estaba por debajo de lo esperado en cuanto a aspecto y cantidad; posicionaba la hoja en ángulo y rotaba frecuentemente su cuerpo o el material al escribir. Una razón detrás de esta problemática podría relacionarse a un pobre procesamiento vestibular impactando negativamente en el desarrollo de la coordinación bilateral, el cruce de la línea media, movimientos oculares y control postural, también aspectos influyentes para la escritura. Benjamín se distraía con los estímulos provenientes del afuera debido a una híper-respuesta visual y auditiva que dificultan la habilidad de filtrar sensaciones y atender sólo a aquellas relevantes. Esta dificultad era mucho más evidente en ambientes con más estímulos como la escuela. Por último, no era muy habilidoso para los deportes de equipo, sobre todo aquellos con pelota; y adoptaba una actitud pasiva apoyándose frecuentemente en muebles y objetos. Esto podría deberse a su pobre control postural, coordinación motora bilateral y planeamiento motor de actividades de anticipación (feedforward), a causa de un pobre procesamiento propioceptivo y vestibular.

\section{Caso César}

\section{Perfil Ocupacional}

César era un niño de 6 años y 1 mes al momento de la evaluación. Vivía con sus padres y cursaba el primer grado en una escuela de Paris. Sus padres y maestros lo describían como una niño sensible, creativo e imaginativo con buenas habilidades de lenguaje.

César nació luego de un embarazo y parto sin complicaciones, cumplió las pautas iniciales de desarrollo madurativo a edades esperadas, y no tenía antecedentes médicos relevantes.
Relación entre resultados de la evaluación y desempeño ocupacional

César demostraba dificultades para mantener una posición sedente estable, cambiaba frecuentemente de postura, y ocasionalmente se caía de la silla. Esto podría relacionarse a un ineficiente control postural debido a problemas de procesamiento propioceptivo y vestibular. Asimismo, ejercía demasiada fuerza al escribir, frecuentemente rompía la hoja y cambiaba constantemente la forma de tomar el lápiz. Tenía dificultades con los botones, cierres, ponerse las medias y zapatos y atar sus cordones. Estas dificultades podrían estar causadas por un pobre procesamiento táctil-propioceptivo que afecta el planeamiento, coordinación y destreza motora fina. Además, su pobre procesamiento vestibular-ocular y dificultades en la percepción visual podrían influir en aspectos de la escritura como el posicionamiento y orientación en la hoja. Por otro lado, los padres del niño estaban preocupados ya que veían que tenía miedo de ciertos movimientos y actividades, y por ende no participaba de ellas, por ejemplo, los juegos de plaza. Tampoco participaba de las clases de educación física en la escuela y tenía dificultades para realizar deportes, sobre todo aquellos con pelota. Esto podría deberse a un pobre procesamiento propioceptivo y vestibular que impacta en la respuesta afectiva al movimiento, el control postural, coordinación motora bilateral y planeamiento motor.

\section{Intervención de Terapia Ocupacional}

En la Tabla 4 se puede observar un esquema global de planificación e intervención. 
TABLA 3 EVALUACIÓN DE COMPONENTES DE DESEMPEÑO

\begin{tabular}{|c|c|c|}
\hline Evaluación & Área de dificultad & Desempeño \\
\hline \multirow{4}{*}{ SPM Hogar } & Tacto & Algunos Problemas ( $\mathrm{T}=63$ ) \\
\hline & Conciencia corporal & Algunos Problemas $(\mathrm{T}=65)$ \\
\hline & Movimiento y equilibrio & Algunos Problemas $(\mathrm{T}=68)$ \\
\hline & Planeamiento motor e ideación & Algunos Problemas $(\mathrm{T}=67)$ \\
\hline \multirow{4}{*}{ SPM Escuela } & Tacto & Algunos Problemas $(\mathrm{T}=67)$ \\
\hline & Conciencia corporal & Algunos Problemas $(\mathrm{T}=66)$ \\
\hline & Movimiento y equilibrio & Algunos Problemas $(\mathrm{T}=64)$ \\
\hline & Planeamiento motor e ideación & Diferencia Definitiva $(\mathrm{T}=70)$ \\
\hline \multirow{3}{*}{ BOT-2 } & Precisión motora fina & Debajo del promedio $(\mathrm{SS}=8)$ \\
\hline & Coordinación de E.S & Debajo del promedio $(\mathrm{SS}=8)$ \\
\hline & Coordinación Bilateral & Debajo del promedio $(\mathrm{SS}=9)$ \\
\hline \multirow{2}{*}{ DTVP-3 } & Coordinación ojo-mano & Pobre $(\mathrm{SS}=6)$ \\
\hline & Figura-Fondo & Pobre (SS=5) \\
\hline \multirow{7}{*}{ Observaciones Clínicas } & Toque secuencial de dedos & \\
\hline & Movimientos Oculares & \\
\hline & Posiciones anti-gravitatorias & \\
\hline & Estabilidad articular proximal & \\
\hline & Control postural con ojos cerrados & \\
\hline & Miedo al movimiento & \\
\hline & Localización y discriminación táctil & \\
\hline
\end{tabular}


TABLA 4. INTERVENCIÓN DE TERAPIA OCUPACIONAL

\section{Plan de intervención}

El marco teórico utilizado principalmente fue el de Integración Sensorial. A continuación, se brindan ejemplos específicos del uso de actividades sensoriales y motoras adaptadas individualmente que fueron co-creadas por el profesional y el niño de acuerdo con las necesidades de éste, contextualizadas en el juego y continuamente ajustadas y adaptadas por el profesional para proporcionar un nivel de desafío que aumente gradualmente la complejidad del desempeño del niño (Watling et al., 2018)

Si bien la integración sensorial fue el marco principal de intervención, se debe tratar al niño en su totalidad en diferentes entornos; para ello, a menudo se requieren múltiples enfoques de intervención. Se mencionan asimismo ciertas estrategias y enfoques complementarios utilizados en cada caso (Reynolds, et al., 2017)

\section{Revisión de intervención}

El proceso de intervención fue revisado mediante reuniones con los padres cada dos meses y comunicación constante con las maestras. Asimismo, padres y maestros colaboraron activamente para la implementación de estrategias y adaptaciones sugeridas por el terapeuta. La duración media de la intervención fue de 27 sesiones individuales de 45 minutos, realizadas una vez por semana en el transcurso del año lectivo. Se realizo además una re-evaluación durante el proceso terapéutico que permitió al terapeuta reajustar sus objetivos en función de estos resultados preliminares. Por motivos de síntesis de resultados tales datos no pudieron ser incluidos en este informe.

\begin{tabular}{|c|c|}
\hline \multicolumn{2}{|r|}{ Ana } \\
\hline $\begin{array}{l}\text { Actividad de } \\
\text { Integración } \\
\text { Sensorial }\end{array}$ & $\begin{array}{l}\text { Circuito de Obstáculos: en un extremo del gimnasio se colocan un recipiente con canicas } \\
\text { de agua y figuras geométricas escondidas dentro, en el otro extremo se coloca una bolsa } \\
\text { opaca con las mismas figuras geométricas escondidas. En el espacio del medio se diseña } \\
\text { junto al niño una ruta con obstáculos. Éstos suelen incluir saltar de un lugar a otro, gatear } \\
\text { por lugares estrechos, caminar por superficies inestables, trepar a distintos materiales, } \\
\text { entre otros. El niño debe meter la mano en el recipiente y encontrar cualquier figura sin } \\
\text { apoyo visual, luego realizar la ruta hasta el otro extremo, meter la mano en la bolsa y sólo } \\
\text { utilizando el tacto encontrar la figura correspondiente. }\end{array}$ \\
\hline Propósito & $\begin{array}{l}\text { - Brindar alta intensidad de input somato sensorial } \\
\text { - } \text { Desafiar el control postural y planeamiento motor } \\
\text { - Favorecer la discriminación táctil }\end{array}$ \\
\hline Variaciones & $\begin{array}{l}\text { Al principio se colocaban figuras muy distintas entre sí, como circulo y cuadrado. Luego se } \\
\text { utilizaban figuras con diferencias sutiles, como cuadrado y rectángulo. También se realizó } \\
\text { esta actividad emparejando texturas. Los obstáculos de la ruta fueron incrementando en } \\
\text { dificultad y la intervención del terapeuta en la realización del circuito fue cada vez menor. } \\
\text { Se realizó el circuito con ojos abiertos y cerrados; parado, en cuatro patas o reptando. }\end{array}$ \\
\hline $\begin{array}{l}\text { Otras } \\
\text { intervenciones }\end{array}$ & $\begin{array}{l}\text { - Se recomendaron recreos de movimiento frecuentes en el hogar y la escuela con el fin } \\
\text { de brindar input vestibular y propioceptivo durante el día. } \\
\text { - Se recomendó el uso de cepillo de dientes vibratorio para brindar más información } \\
\text { táctil en el área oral. } \\
\text { - Se acordó que Ana podría masticar chicle en clase con el fin de reemplazar otros } \\
\text { comportamientos relacionados a la búsqueda de información propioceptiva menos } \\
\text { adecuados. } \\
\text { - Se utilizó el programa "Zonas de regulación" para trabajar la regulación emocional y se } \\
\text { derivó a psicología para trabajar el manejo de stress, ansiedad y autoestima. }\end{array}$ \\
\hline
\end{tabular}




\begin{tabular}{|c|c|}
\hline \multicolumn{2}{|r|}{ Benjamín } \\
\hline $\begin{array}{l}\text { Actividad de } \\
\text { Integración } \\
\text { Sensorial }\end{array}$ & $\begin{array}{l}\text { Juego de puntos: niño en prono en la hamaca de plataforma debe tirar de una soga para } \\
\text { alcanzar una pelota sostenida en lo alto por el terapeuta. El niño se impulsa en la hamaca } \\
\text { tirando de la soga y debe arrojar la pelota a distintos aros colocados en el piso a diferentes } \\
\text { distancias. A mayor distancia mayor puntuación. }\end{array}$ \\
\hline Propósito & $\begin{array}{l}\text { - } \text { Brindar input vestibular-propioceptivo } \\
\text { - Desafiar el tono extensor } \\
\text { - Desafiar el planeamiento motor (actividad de feedforward) } \\
\text { Desafiar la coordinación motora bilateral }\end{array}$ \\
\hline Variaciones & $\begin{array}{l}\text { Se utilizaron pelotas de distinto peso. Se realizó la actividad en distintas hamacas y en dis- } \\
\text { tintas posiciones (sentado, arrodillado, prono). Finalmente, el objetivo fue tirar la pelota a } \\
\text { través de un aro en movimiento. }\end{array}$ \\
\hline $\begin{array}{l}\text { Otras } \\
\text { intervenciones }\end{array}$ & $\begin{array}{l}\text { - Recreos de movimiento frecuentes } \\
\text { - Se acordó con la maestra que Benjamín podría realizar algunas actividades en la clase } \\
\text { en posición de parado o acostado en el suelo. } \\
\text { - Se ubicó a Benjamín en un lugar de la clase donde había menos distracciones visuales } \\
\text { y auditivas. }\end{array}$ \\
\hline \multicolumn{2}{|r|}{ César } \\
\hline \multirow{2}{*}{$\begin{array}{l}\text { Actividad de } \\
\text { Integración } \\
\text { Sensorial }\end{array}$} & $\begin{array}{l}\text { Tormenta en el barco: niño sentado en la hamaca de plataforma colocada muy cerca del } \\
\text { suelo. El terapeuta realiza movimientos suaves y rítmicos de la hamaca simulando el movi- } \\
\text { miento de un barco en el agua. El terapeuta anticipa una tormenta y realiza movimientos } \\
\text { para que el niño caiga de la hamaca a la colchoneta. Al caer, se presiona al niño con un } \\
\text { almohadón simulando las olas que rompen. }\end{array}$ \\
\hline & $\begin{array}{l}\text { Búsqueda del tesoro de pókemon: Se colocan obstáculos en todo el gimnasio y se ponen } \\
\text { pequeños muñecos (pókemons) "escondidos" en el espacio a diferentes alturas y distan- } \\
\text { cias. El niño tiene que sortear los obstáculos para capturar los pókemons y llevarlos a través } \\
\text { de una ruta a su guarida. }\end{array}$ \\
\hline Propósito & $\begin{array}{l}\text { - Brindar input vestibular apareado con alta intensidad de input propioceptivo y de pre- } \\
\text { sión profunda. } \\
\text { - } \text { Favorecer la tolerancia al movimiento } \\
\text { - Desafiar la percepción visual figura-fondo } \\
\text { Desafiar el control postural y planeamiento motor }\end{array}$ \\
\hline & $\begin{array}{l}\text { 1. A medida que el niño se siente más cómodo con el movimiento, se va subiendo la } \\
\text { altura de la hamaca y realizando movimientos mas bruscos. }\end{array}$ \\
\hline Variaciones & $\begin{array}{l}\text { 2. Se fue incrementando la dificultad de visualización del objeto por ejemplo colocando } \\
\text { un pókemon en un fondo del mismo color. Asimismo, los obstáculos que había que } \\
\text { sortear para llegar a ellos fueron incrementando en dificultad, por ejemplo, colocar el } \\
\text { objeto a mayor altura en la pared de escalar. }\end{array}$ \\
\hline $\begin{array}{l}\text { Otras } \\
\text { intervenciones }\end{array}$ & $\begin{array}{l}\text { - Recreos de movimiento frecuentes } \\
\text { - Modificación de la ubicación de César en la clase. Se lo ubicó de manera que este } \\
\text { cabentado al pizarrón y no de perfil, para evitar realizar grandes movimientos de la } \\
\text { - Se utilizó una aplicación diseñada para trabajar procesamiento visual. } \\
\text { - Se utilizó un guante de escritura diseñado para favorecer una buena toma del lápiz al } \\
\text { brindar mayor input táctil y propioceptivo. }\end{array}$ \\
\hline
\end{tabular}




\section{ReSUltados}

Resultados de desempeño funcional: Los tres niños. demostraron mejoras significativas en la calidad de su escritura, reportado así por sus padres y maestros y corroborado con muestras de escritura.

Asimismo, los padres y maestros refirieron tener un mejor entendimiento de las problemáticas de los niños y mayor habilidad para implementar estrategias y actividades que apoyen el desempeño. Por ejemplo, los padres de Ana comenzaron a incorporar más actividad física en la vida familiar y a anticiparse a situaciones que podían generar stress para Ana, por ejemplo, Ilevándola a visitar previamente el lugar donde se celebraría un cumpleaños.

Por último, los tres niños aumentaron su participación en deportes y actividades de motricidad gruesa. Por ejemplo, César aceptó ser inscripto en la actividad extracurricular llamada "multideportiva".

En el caso de Ana, sus padres vieron un impacto positivo en la autoestima de su hija, en su manejo durante la alimentación y en sus niveles de energía. En un email la madre dijo: "Ojalá lo hubiera grabado, pero ayer vi a Ana correr y jugar en un patio con sus amigos, que son cuatro niños muy atléticos. Se subió a la pared de escalar, se deslizó por el tobogán y corrió con ellos. La alegría en su rostro no tenía precio. Habrías estado muy orgulloso de ella."

En el caso de Benjamín, los padres de benjamín mencionaron que la actividad de alimentación ya no era un problema y que observaban grandes mejoras en la coordinación motora de su hijo. En una reunión el padre dijo: "Su escritura ha mejorado muchísimo y ahora le gusta hacer carteles para la casa en los que escribe en espacios muy chicos. Ya no mancha todo cuando come y ahora es más rápido que su hermana".

En el caso de Cesar, el niño logró la independencia completa en el vestido. Su maestra mencionó que utilizaba una pinza trípode sin necesidad de recordatorios. Su padre en una reunión dijo que lo veía menos miedoso y más dispuesto a participar en juegos nuevos.

Resultados de componentes del desempeño: Las siguientes figuras muestran los resultados estandarizados obtenidos por cada niño pre y post intervención.

FIGURA 1 COMPARACIÓN DE RESULTADOS PRE Y POST INTERVENCIÓN DEL BOT-2

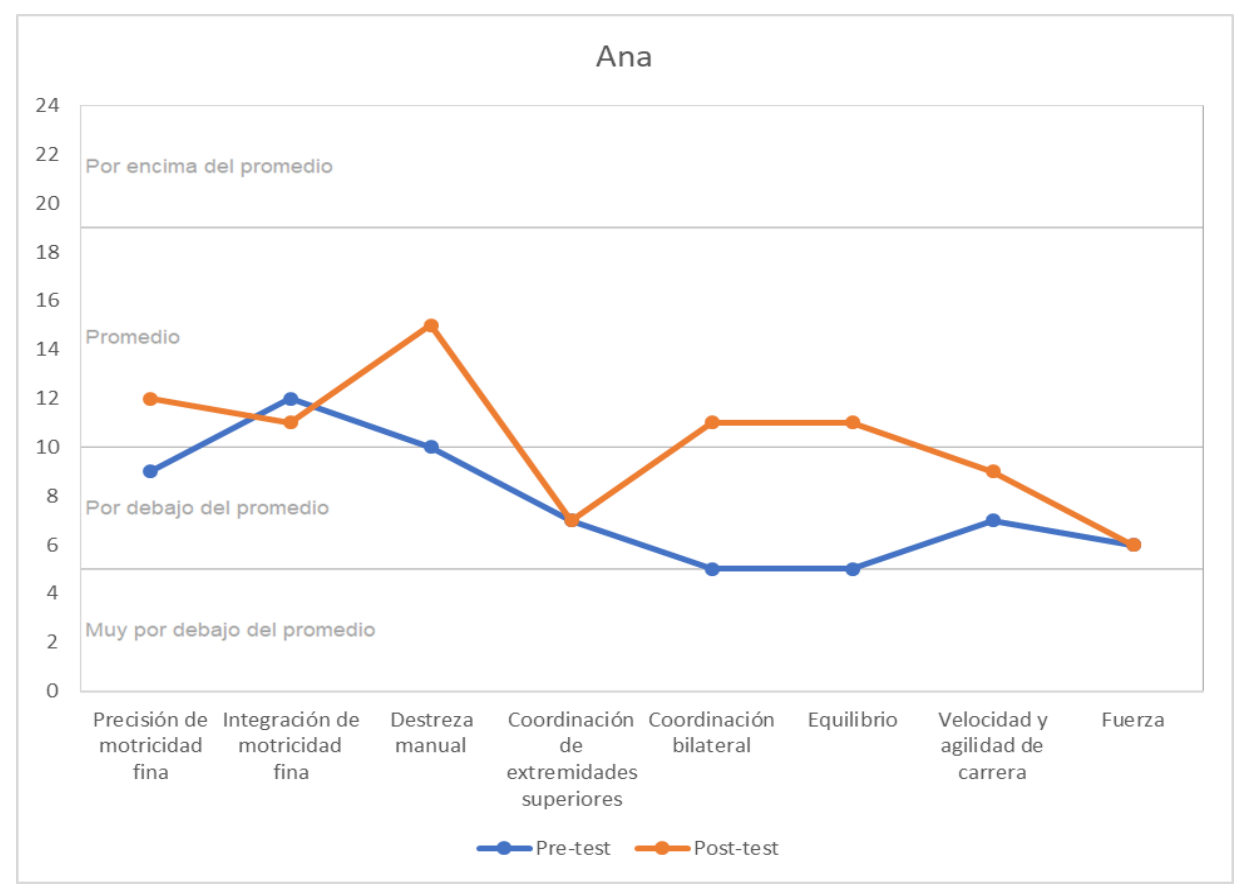



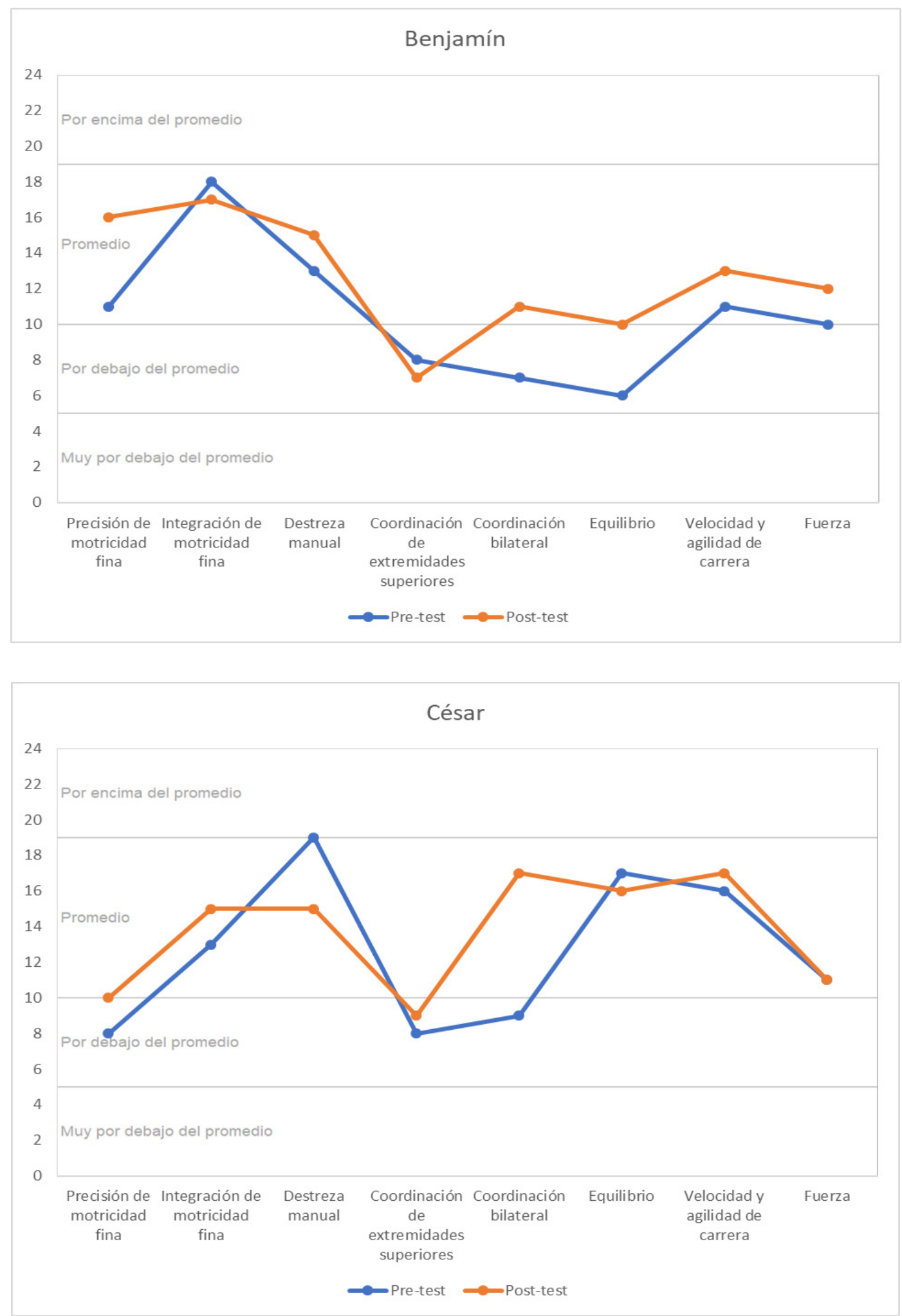
Todos los niños obtuvieron mayores puntuaciones en las subpruebas de "Precisión de motricidad fina", "Coordinación bilateral", "Velocidad y agilidad de carrera" y "Fuerza". Ana y Benjamín también obtuvieron mayores puntuaciones en las pruebas de "Destreza manual" y "Equilibrio". Ana y César también obtuvieron mayores puntuaciones en la escala de "Coordinación de las extremidades superiores".

FIGURA 2 COMPARACIÓN DE RESULTADOS PRE Y POST INTERVENCIÓN DEL DTVP-3

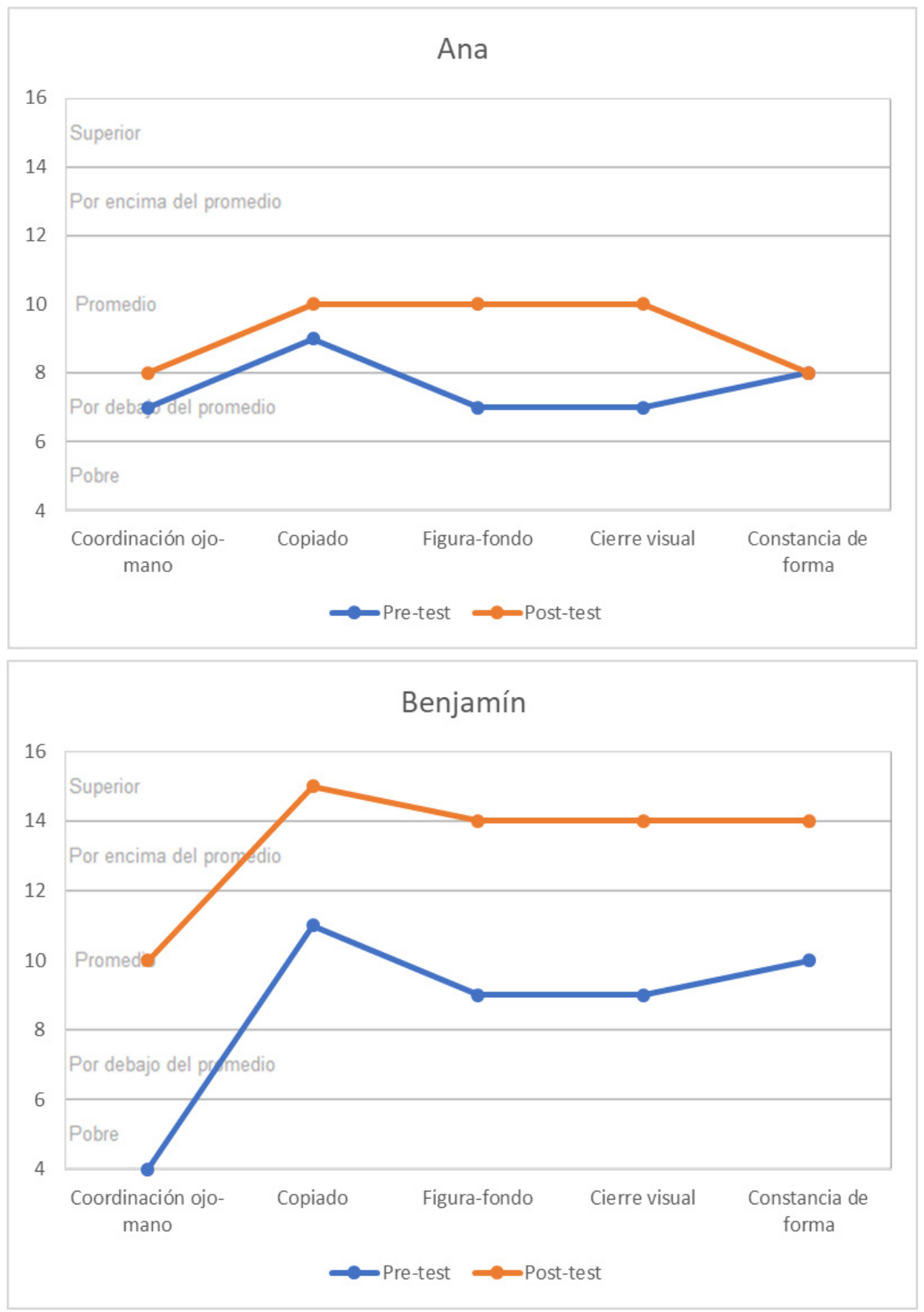




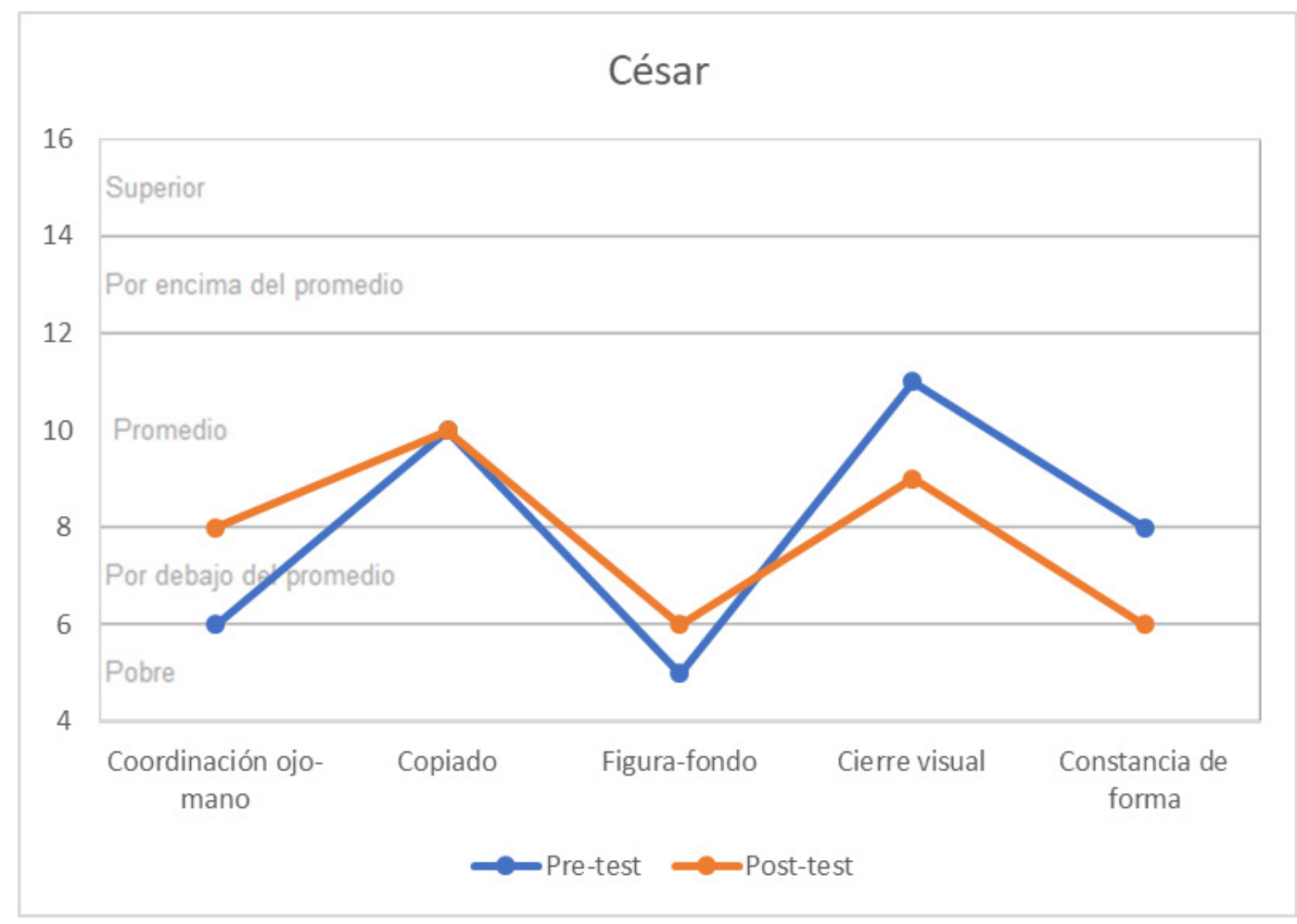

Todos los participantes obtuvieron mayores puntuaciones en las escalas de "Coordinación ojo-mano" y "Figura-fondo". Ana y Benjamín también obtuvieron mayores puntuaciones en la escala de "Cierre visual y constancia de la forma".

\section{Discusión}

El uso de integración sensorial como intervención en terapia ocupacional es cada vez más popular y un número cada vez mayor de estudios está investigando la eficacia de este enfoque (Schoen, Lane, Mailloux, May-Benson, Parham, Smith-Roley \& Schaaf 2019). Asimismo, la teoría y práctica de integración sensorial continúa evolucionando a medida que surge una mayor comprensión de la neurobiología del comportamiento humano y de cómo las diferencias en el funcionamiento neuronal afectan a la participación y desempeño ocupacional (Lane, Mailloux, Schoen, Bundy, May-Benson, Parham, Smith-Roley, Schaaf 2019). Los resultados de este estudio se adhieren a la literatura en el sentido de que la terapia ocupacional bajo un marco de integración sensorial parece ser un método efectivo para mejorar las habilidades de desempeño y la participación funcional de los niños con desafíos de procesamiento sensorial. Asimismo, el trabajo colaborativo entre terapeuta, maestros y padres tuvo un rol clave en la obtención de estos resultados.

Estudios futuros sobre el desempeño ocupacional de estos niños para determinar si los efectos del tratamiento fueron sostenidos en el tiempo, podrían contribuir a confirmar la hipótesis planteada de que un mayor conocimiento y entendimiento tanto por padres como maestros; así como contar con la posibilidad de consultar de forma inmediata con un profesional, son componentes claves para la identificación de problemas de procesamiento sensorial, posibilitando así la intervención tempranas y prevención de mayores dificultades futuras.

A pesar de la conocida incidencia de problemas de procesamiento sensorial en niños que, por lo demás, tienen un desarrollo típico, y de los efectos negativos establecidos que éstos pueden tener en el desempeño ocupacional, se han realizado investigaciones limitadas centradas exclusivamente en esta población. Gran parte 
de la investigación hasta la fecha se ha centrado en los individuos con TEA; otras poblaciones requieren un estudio más profundo (Watling et al., 2018).

Se necesitan, asimismo, más estudios descriptivos que examinen directamente la relación entre el procesamiento sensorial y el desempeño ocupacional; ya que la literatura a menudo no relaciona los problemas sensoriales con las dificultades de desempeño (Koenig \& Rudney, 2010)

Este estudio de casos múltiples apoya el uso de la intervención de integración sensorial en niños de desarrollo típico con dificultades de procesamiento sensorial. Sin embargo, revela la necesidad y el llamado a los terapeutas ocupacionales a explorar más en profundidad estas áreas de investigación con el fin de establecer las mejores prácticas para ayudar a estos niños a alcanzar su pleno potencial.

Por último, se debe destacar que estos niños concurrían a una escuela privada y el cargo de terapia ocupacional en escuelas del estado es poco común en Francia. Como se ha discutido en este estudio, el rol del terapeuta ocupacional en la escuela puede favorecer el desempeño funcional de niños que presentan dificultades del procesamiento sensorial, entre otros. Por ende, es imperativo generar evidencia que impulse la incorporación de los servicios de Terapia Ocupacional en todas las escuelas.

Las limitaciones de este estudio se relacionan principalmente a la pequeñez de la muestra y a la utilización de ciertos instrumentos con poblaciones para los que no fueron validados. Asimismo, la misma terapista ocupacional realizó la evaluación e intervención de terapia ocupacional, así como la escritura de este estudio y la selección de los casos según sus características. Por ende, los resultados descritos y el análisis de este estudio deben interpretarse con total cautela por posibles sesgos.

\section{RefERENCIAS BibLIOGRÁFICAS}

Ayres, A. J. (1972). Sensory Integration and Learning Disorders. Los Angeles: Western Psychological Services.

Blanche, E. I. (2010). Observations Based on Sensory Integration Theory. Torrance: Pediatric Therapy Network.
Bruininks, R. H., \& Bruininks, B. D. (2005). Bruininks-Oseretsky Test of Motor Proficiency $\mid$ Second Edition (BOT-2). Bloomington: Pearson.

Clark, G., Watling, R., Parham, L., \& Schaaf, R. (2019). Occupational Therapy Interventions for Children and Youth With Challenges in Sensory Integration and Sensory Processing: A School-Based Practice Case Example. The American Journal of Occupational Therapy.

Grajo, L., Candler, C., \& Sarafian, A. (2020). Interventions Within the Scope of Occupational Therapy to Improve Children's Academic Participation: A Systematic Review. The American Journal of Occupational Therapy.

Lane, S. J., Mailloux, Z., Schoen, S., Bundy, A., May-Benson, T. A., Parham , L. D., .. Schaaf, R. C. (2019). Neural Foundations of Ayres Sensory Integration ${ }^{\circledR}$. Brain Sciences.

Reynolds, S., Glennon, T. J., Ausderau, K., Bendixen, R. M., Miller Kuhaneck, H., Pfeiffer, B., . . Bodison, S. C. (2017). Using a Multifaceted Approach to Working With Children Who Have Differences in Sensory Processing and Integration. American Journal of Occupational Therapy.

Hammill, D. D., Pearson, N. A., \& Voress, J. K. (2013). Developmental Test of Visual Perception - Third Edition (DTVP-3). . Pre.ed.

Parham, L. D., \& Ecker, C. (2007). Sensory Processing Measure (SPM) Home Form. Los Angeles: Western Psychological Services.

Parham, L. D., \& Ecker, C. (2007). Sensory Processing Measure (SPM)Main Classroom Form. Los Angeles: Western Psychological Services.

Pfeiffer, B., May-Benson, T. A., \& Bodison, S. C. (2018). GUEST EDITORIAL - State of the Science of Sensory Integration Research With Children and Youth. American Journal of Occupational Therapy.

Smith-Roley, S., Bissell, J., \& Clark, G. F. (2009). Providing Occupational Therapy Using Sensory Integration Theory and Methods in SchoolBased Practice. The American Journal of Occupational Therapy.

Smith-Roley, S., Clark, G. F., \& Bissell, J. (2003). Applying Sensory Integration Framework in Educationally Related Occupational Therapy Practice (2003 Statement). American Journal of Occupational Therapy,.

Schaaf, R. C., \& Smith-Roley, S. (2006). Sensory Integration: Applying Clinical Reasoning to Practice with Diverse Populations. Austin, Texas: Pro.ed.

Schoen, S. A., Lane, S. J., Mailloux, Z., May-Benson, T., Parham, D. L., Smith-Roley, S., \& Schaaf, R. C. (2019). A Systematic Review of Ayres Sensory Integration Intervention for Children with Autism. Autism Research.

Watling, R., Kuhaneck, M. H., Parham, D. L., \& Schaaf, R. (2018). Occupational Therapy Practice Guidelines for Children and Youth With Challenges in Sensory Integration and Sensory Processing. Bethesda: MD: AOTA Press.

Koenig, K. P., \& Rudney, S. G. (2010). Performance Challenges for Children and Adolescents With Difficulty Processing and Integrating Sensory Information: A Systematic Review. American Journal of Occupational Therapy. 